\title{
The Chiral Determinant and the Eta Invariant
}

\author{
S. Della Pietra ${ }^{1,2, \star, \star \star}$, V. Della Pietra ${ }^{1, \star}$, and L. Alvarez-Gaumé ${ }^{1, \star, \star \star \star}$ \\ ${ }^{1}$ Lyman Laboratory of Physics, Harvard University, Cambridge, MA 02138, USA \\ 2 Theory Group, Physics Department, University of Texas, Austin, TX 78712, USA
}

\begin{abstract}
For $\left\{\partial_{y}\right\}, y \in \mathbb{R}$, a one parameter family of invertible Weyl operators of possibly non-zero index acting on spinors over an even dimensional compact manifold $X$, we express the phase of the chiral determinant $\operatorname{det} \partial_{-\infty}^{\dagger} \partial_{\infty}$ in terms of the $\eta$ invariant of a Dirac operator acting on spinors over $\mathbb{R} \times X$.
\end{abstract}

\section{Statement of Results}

Let $X$ be a compact spin manifold of even dimension with spin bundle $S=S_{+} \oplus S_{-}$ $\rightarrow X$ and let $E \rightarrow X$ be a hermitian vector bundle over $X$. Let $\bar{S}=\mathbb{R} \times S, \bar{E}=\mathbb{R} \times S$ be the pullbacks of $S$ and $E$ to $\mathbb{R} \times X$ with the pull-back hermitian inner products, and let $\nabla^{\bar{E}}$ be a connection on $\bar{E}$. Thus $\nabla^{\bar{E}}=d_{\mathbb{R}}+\theta+\nabla_{(\cdot)}^{E}$, where $\theta \in \Omega^{1}(\mathbb{R}) \otimes C^{\infty}(X$, End $E)$, and for each $u \in \mathbb{R}, \nabla_{u}^{E}$ is a connection on $E \rightarrow X$.

For $\quad u \in \mathbb{R}$, let $\quad \partial_{u}: C^{\infty}\left(X, S_{+} \otimes E\right) \mapsto C^{\infty}\left(X, S_{-} \otimes E\right) \quad$ and $D_{u}: C^{\infty}(X, S \otimes E) \mapsto C^{\infty}(X, S \otimes E)$ be the Weyl and Dirac operators coupled to the metric on $X$ and the connection $\nabla_{u}^{E}$ on $E$. In the decomposition defined by $S=S_{+} \oplus S_{-}, D_{u}=\left(\partial_{u} \partial_{u}^{\dagger}\right)$. Let $H$ be the formally self-adjoint Dirac operator on $L^{2}(\mathbb{R} \times X, \bar{S} \otimes \bar{E})$ coupled to the connection $\nabla^{\bar{E}}$ on $\bar{E}$ and the product metric on $\mathbb{R} \times X$. Thus $H=i \Gamma\left(\frac{\partial}{\partial u}+\theta\left(\frac{\partial}{\partial u}\right)\right)+D_{(\cdot)}$, where $\Gamma$ is the endomorphism of $S$ with $\Gamma= \pm 1$ on $S_{ \pm}$.

\section{Assume}

1. For all $u \in \mathbb{R}, \partial_{-\infty}^{\dagger} \partial_{u}$ is invertible.

2. For $|u|$ large, $\theta=0$ and $d \nabla^{E} / d u=0$.

Condition 1 implies that for all $u, \operatorname{Ker} \partial_{u}=0$ and $\operatorname{Ker} \partial_{u}^{\dagger}$ is a finite dimensional complement in $L^{2}$ of $\operatorname{Im} \partial_{-\infty}$. Condition 2 implies that for $|u|$ large, $\partial_{u}$ is independent of $u$ and $H$ is invariant under translations in the $\mathbb{R}$ direction.

\footnotetext{
* Supported in part by NSF Grant No. PHY-82-15249

$\star \star$ Supported in part by NSF Grant PHY 8605978 and the Robert A. Welch Foundation $\star \star \star$ Present address: Theory Division, CERN, CH-1211 Geneva 23, Switzerland
} 
Let $P_{-\infty}$ be orthogonal projection in $L^{2}(X, S \otimes E)$ onto $\operatorname{Im} D_{-\infty}$, and let $\bar{P}_{-\infty}$ be the translation invariant operator on $L^{2}(\mathbb{R} \times X, \bar{S} \otimes \bar{E})$ induced by $P_{-\infty}$. Let $\bar{V}=\operatorname{Im} \bar{P}_{-\infty}$ with the induced inner product, and let $\left.H\right|_{\bar{V}}$ be the operator on $\bar{V}$ induced by $\bar{P}_{-\infty} H \bar{P}_{-\infty}$ on $L^{2}(\mathbb{R} \times X, \bar{S} \otimes \bar{E})$.

Let $\psi_{ \pm \infty}^{i}, i=1, \ldots, \operatorname{dim} \operatorname{Ker} \partial^{\dagger}$ be bases for $\operatorname{Ker} \partial_{ \pm \infty}^{\dagger}$ respectively.

We will prove

Theorem (1.1). Under the assumptions above,

$$
\begin{aligned}
& \frac{\operatorname{det} \partial_{-\infty}^{\dagger} \partial_{\infty}}{\left|\operatorname{det}\left\langle\psi_{\infty}, \psi \psi_{-\infty}\right\rangle\right|}=\left(\frac{\operatorname{det} \partial_{\infty}^{\dagger} \partial_{\infty}}{\operatorname{det}\left\langle\psi_{\infty}, \psi_{\infty}\right\rangle}\right)^{1 / 2}\left(\frac{\operatorname{det} \partial_{-\infty}^{\dagger} \partial_{-\infty}}{\operatorname{det}\left\langle\psi-\infty, \psi_{-\infty}\right\rangle}\right)^{1 / 2} \\
& \quad \times \exp 2 \pi i\left(\xi\left(\left.H\right|_{\bar{V}}\right)-Q\left(\nabla^{\bar{E}}\right)\right) \exp \int_{\mathbb{R}} \operatorname{Tr}\left(1-P_{-\infty}\right) \theta \exp B\left(\nabla_{-\infty}^{E}, \nabla_{\infty}^{E}, g\right),
\end{aligned}
$$

where

$$
\begin{aligned}
\operatorname{det} \partial_{ \pm \infty}^{\dagger} \partial_{ \pm \infty} & =\exp -\lim _{z \rightarrow 0} \frac{d}{d z} \operatorname{Tr}\left(\partial_{ \pm \infty}^{\dagger} \partial_{ \pm \infty}\right)^{-z}, \\
\xi\left(\left.H\right|_{\bar{V}}\right) & =\frac{1}{2}\left(\eta\left(\left.H\right|_{\bar{V}}\right)+\left.\operatorname{dim} \operatorname{Ker} H\right|_{\bar{V}}\right), \\
\eta\left(\left.H\right|_{\bar{V}}\right) & =\left.\lim _{z \rightarrow 0} \lim _{\phi \rightarrow 1} \operatorname{Tr} \phi H\right|_{\bar{V}}\left(\left.H\right|_{\bar{V}}{ }^{2}\right)^{-z-1 / 2}, \\
Q\left(\nabla^{\bar{E}}\right) & =\int_{t \in[0,1] \mathbb{R} \times X} \int_{X} \hat{A}\left(\mathscr{R}_{g}\right) \operatorname{ch}\left(\mathscr{F}_{\nabla[0,1] \times \bar{E}}\right),
\end{aligned}
$$

with

$$
\nabla^{[0,1] \times \bar{E}}=d t \frac{\partial}{\partial t}+t \nabla^{\bar{E}}+(1-t) \nabla_{-\infty}^{E}
$$

and $B\left(\nabla_{-\infty}^{E}, \nabla_{\infty}^{E}, g\right)$ is a local functional of the connections $\nabla_{ \pm \infty}^{E}$ and the metric g. (See below for a precise statement.)

In the definitions of det and $\eta$, the complex powers of $\partial_{ \pm \infty}^{\dagger} \partial_{\infty}$ and $\left.H\right|_{\vec{V}}{ }^{2}$ are defined by contour integration as in $[7,8] . \phi$ is a smooth function on $\mathbb{R}$ of compact support acting as a multiplication operator on $L^{2}(\mathbb{R} \times X, \bar{S} \otimes \bar{E})$, and $\phi \rightarrow 1$ is taken through a pointwise increasing sequence of such $\phi . \lim _{z \rightarrow 0}$ is understood as the value at $z=0$ of the analytic continuation of a function which is analytic for $\operatorname{Re} z \gg 0$.

In the definition of $Q, g$ is the ( $\mathbb{R}$-independent) metric on $X, \mathscr{R}_{g}$ is the curvature of the Levi-Civita connection for $g$, and $\nabla^{[0,1] \times \bar{E}}$ is interpreted as a connection on the pull-back of $\bar{E}$ to a bundle over $[0,1] \times \mathbb{R} \times X$, and $\mathscr{F}_{\nabla^{[0,1]} \times \bar{E}}$ is its curvature:

$$
\mathscr{F}_{\nabla^{[0,1] \times \bar{E}}}=\mathscr{F}_{\nabla_{\bar{E}}}+d t\left(\nabla^{\bar{E}}-\nabla_{-\infty}^{E}\right) \in \Omega^{2}([0,1] \times \mathbb{R} \times X, \operatorname{End}([0,1] \times \bar{E})) .
$$

$\widehat{A}$ and ch are the polynomials

$$
\hat{A}(\mathscr{R})=\sqrt{\operatorname{det}\left(\frac{\mathscr{R} / 4 \pi}{\sinh \mathscr{R} / 4 \pi}\right)}, \quad \operatorname{ch}(\mathscr{F})=\operatorname{tr} \exp i \mathscr{F} / 2 \pi .
$$

Finally, $B\left(\nabla_{-\infty}^{E}, \nabla_{+\infty}^{E}, g\right)$ is local in the following sense: 
There is an explicitly computable universal polynomial $[B]$ in $(\operatorname{det} g)^{-1}$, the components of $g$, and the components of the multiple covariant derivatives (in the $X$ directions) of $\mathscr{R}_{g}, \mathscr{F}_{\nabla E_{\infty}}$, and $\nabla_{+\infty}^{E}-\nabla_{-\infty}^{E}$, such that

$$
B\left(\nabla_{-\infty}^{E}, \nabla_{+\infty}^{E}, g\right)=\int_{X}[B]\left(g, \nabla^{*} \mathscr{R}_{g}, \nabla^{*} \mathscr{F}_{\nabla_{-}}, \nabla^{*}\left(\nabla_{\infty}^{E}-\nabla_{-\infty}^{E}\right)\right)|d x| .
$$

Here $\mathscr{F}_{\nabla \underline{E}_{\infty}}$ is the curvature of $\nabla_{-\infty}^{E}$, and $\nabla^{*}$ denotes multiple covariant derivatives with respect to the connection determined by $g$ and $\nabla_{-\infty}^{E}$. We investigate the exact form of $B\left(\nabla_{-\infty}^{E}, \nabla_{\infty}^{E}, g\right)$ in Sect. 4, where we find

Proposition (1.5). If $\operatorname{dim} X=2$ or 4 , then $\operatorname{Im} B=0$.

We conjecture that $\operatorname{Im} B=0$ generally.

In the language of renormalization, the functional $[B]$ represents a "local counterterm" depending on the data $\nabla_{ \pm \infty}^{E}$ and $g$ over $X$. Although it can be calculated explicitly, it can be often be ignored. In contrast, although $Q$ is a local functional of $\nabla^{\bar{E}}$, it depends not only on $\nabla_{ \pm \infty}^{E}$, but also on $\theta$ and $\nabla_{u}^{E}$ for all $u \in \mathbb{R}$. Thus an exact expression for $Q\left(\nabla^{\vec{E}}\right)$ is relevant.

In a quantum field theory containing gauge fields $A$ and $A^{\prime}$ coupled to positive chirality and negative chirality Weyl Fermions respectively, the quantity $\Gamma\left[A, A^{\prime}\right]$ $=\log \operatorname{det} \partial_{A}^{\dagger} \partial_{A^{\prime}}$ arises as result of a partial integration in the functional integral over the Fermionic fields, and can be interpreted as the effective action for the gauge fields due to the quantum fluctuations of the Fermions. Such determinants can also enter via Faddeev-Popov ghosts and from bosonic integration in String Theory. Theorem (1.1) expresses the real part of $\Gamma\left[A, A^{\prime}\right]$ in terms of the effective actions $\Gamma[A, A]$ and $\Gamma\left[A^{\prime}, A^{\prime}\right]$ of vector-like theories in which the gauge fields are coupled to Dirac Fermions, and it expresses the imaginary part of $\Gamma\left[A, A^{\prime}\right]$ in terms of the local functional $Q\left(\nabla^{\bar{E}}\right)$ and the spectral asymmetry $\eta\left(\left.H\right|_{\bar{V}}\right)$ and zero modes $\left.\operatorname{dim} \operatorname{Ker} H\right|_{\bar{V}}$ of a Dirac operator $\left.H\right|_{\bar{V}}$ in one more dimension. With the exception of the term involving the zero-mode eigenfunctions $\psi$, this expression for $\operatorname{Re} \Gamma\left[A, A^{\prime}\right]$ is formally well known, and the behavior of $\operatorname{Im} \Gamma\left[A, A^{\prime}\right]$ along gauge orbits, that is, its variation under independent gauge transformations of $A$ and $A^{\prime}$, has been investigated extensively in connection with perturbative gauge anomalies [4], and references therein), in which the functional $Q\left(\nabla^{\bar{E}}\right)$ appears, the Witten $S U(2)$ anomaly [15], in which a term analogous to $\operatorname{dim} \operatorname{Ker}\left(\left.H\right|_{\bar{V}}\right)$ appears, and the global gravitational anomalies [16], in which a term analogous to $\eta\left(\left.H\right|_{\bar{V}}\right)$ appears. Theorem (1.1) thus represents a synthesis of these results, extended to describe the complete behavior of $\Gamma\left[A, A^{\prime}\right]$ including its variation across gauge orbits.

\section{Previous Results}

Our proof of Theorem (1.1) is based on some of the results of our previous paper [8]. In this section we briefly review what we will need.

We first generalize the setting above by replacing $\mathbb{R}$ by an arbitrary smooth manifold $Y$. Thus we now let $\bar{E}$ be the pull-back to $Y \times X$ of $E$, let $\nabla^{\bar{E}}=d_{Y}+\theta+\nabla_{(\cdot)}^{E}$ be a connection on $\bar{E}$, and let $\partial_{y}, y \in Y$, be the Weyl operator coupled to the connection $\nabla_{y}^{E}$ and the metric on $X$. In [8] we interpreted these data geometrically within the framework developed by Bismut and Freed for studying families of 
elliptic operators. In the present context this is not necessary and we refer the reader to [8] for a discussion of the geometric setting. We will, however, use the notation of [8]. In particular, let $\nabla \partial=d_{Y} \partial+[\theta, \partial]$.

Fix $y_{0} \in Y$ and assume

$1^{\prime}$. For all $y \in Y, \partial_{y_{0}}^{\dagger} \partial_{y}$ is invertible.

Thus for all $y \in Y$, $\operatorname{Ker} \partial_{y}=0$ and $\operatorname{Ker} \partial_{y_{0}}^{\dagger}$ is a finite dimensional complement in $L^{2}\left(X, S_{-} \otimes E\right)$ of $\operatorname{Im} \partial_{y_{0}}$.

Let $P_{y_{0}}$ be the operator on $L^{2}\left(X, S_{-} \otimes E\right)$ given by orthogonal projection onto $\operatorname{Im} \partial_{y_{0}}$. Then $P_{y_{0}}$ induces an isomorphism $1-P_{y_{0}}: \operatorname{Ker} \partial_{y}^{\dagger} \mapsto \operatorname{Ker} \partial_{y_{0}}^{\dagger}$, and so we can choose bases $\left\{\psi_{y}^{i}\right\}$ for $\operatorname{Ker} \partial_{y}^{\dagger}, i=1,2, \ldots, n=\operatorname{dim} \operatorname{Ker} \partial^{\dagger}, y \in Y$, depending smoothly on $y$.

Define the following functions and one-forms on $Y$ :

$$
\begin{aligned}
\operatorname{det} \partial^{\dagger} \partial= & \exp -\lim _{z \rightarrow 0} \frac{d}{d z} \operatorname{Tr}\left(\partial^{\dagger} \partial\right)^{-z}, \\
\operatorname{det} \partial_{y_{0}}^{\dagger} \partial= & \exp -\lim _{z \rightarrow 0} \frac{d}{d z} \operatorname{Tr}\left(\partial_{y_{0}}^{\dagger} \partial\right)^{-z}, \\
\omega^{\theta}= & \{\text { f.p.a. } z=0\} \operatorname{Tr}\left(\partial^{\dagger} \partial\right)^{-z-1} \partial^{\dagger}(\nabla \partial), \\
\sigma_{0}^{\theta}= & \{\text { f.p.a. } z=0\} \frac{1}{2} \operatorname{Tr}\left(\partial^{\dagger} P_{y_{0}} \partial\right)^{-z-1}\left(\partial^{\dagger} P_{y_{0}}(\nabla \partial)-\left(\nabla \partial^{\dagger}\right) P_{y_{0}} \partial\right), \\
z_{0}^{\theta}= & -\operatorname{Tr}\left(\left(\partial^{\dagger} \partial\right)^{-1} \partial^{\dagger}-\left(\partial_{y_{0}}^{\dagger} \partial\right)^{-1} \partial_{y_{0}}^{\dagger}\right)(d \partial) \\
& +\operatorname{Tr}\left(1-\partial\left(\partial^{\dagger} \partial\right)^{-1} \partial^{\dagger}\right) \theta-\operatorname{Tr}\left(1-P_{y_{0}}\right) \theta, \\
h_{0}= & \left|\operatorname{det}\left\langle\psi, \psi_{y_{0}}\right\rangle\right|^{2} /\left(\operatorname{det}\langle\psi, \psi\rangle \operatorname{det}\left\langle\psi_{y_{0}}, \psi_{y_{0}}\right\rangle\right), \\
J_{y_{0}}= & \lim _{z \rightarrow 0} \operatorname{Tr}\left(\left(\partial^{\dagger} \partial\right)^{-z-1} \partial^{\dagger}-\left(\partial_{y_{0}}^{\dagger} \partial\right)^{-z-1} \partial_{y_{0}}^{\dagger}\right)(d \partial) \\
& -\operatorname{Tr}\left(\left(\partial^{\dagger} \partial\right)^{-1} \partial^{\dagger}-\left(\partial_{y_{0}}^{\dagger} \partial\right)^{-1} \partial_{y_{0}}^{\dagger}\right)(d \partial), \\
L^{\theta}= & \left.\lim _{z \rightarrow 0}\left(\operatorname{Tr}\left(\partial \partial^{\dagger}\right)^{-z} \theta-\operatorname{Tr}\left(\partial^{\dagger} \partial\right)^{-z}\right) \theta\right)+\operatorname{Tr}\left(1-\partial\left(\partial^{\dagger} \partial\right)^{-1} \partial^{\dagger}\right) \theta .
\end{aligned}
$$

The operator complex powers appearing in these expressions are defined by contour integration as we explained in [8] (see also [13,14]). Here we must also deal with the non-self-adjoint operator $\partial_{y_{0}}^{\dagger} \partial_{y}$, but this causes no additional problems since the principal symbol of $\partial_{y_{0}}^{\dagger} \partial$ is strictly positive. It can be shown that the spectrum of $\partial_{y} \partial_{y_{0}}^{\dagger}$ consists of isolated points corresponding to generalized eigenspaces of finite multiplicity, and only a finite number of these points lie in any closed sector not containing the positive real axis.

By the techniques of Seeley as discussed in $[13,14]$ and Appendix C of [7] it can be shown that in the definitions $(2.1)-(2.9)$, the traces involving the operator complex powers are analytic in $z$ for $\operatorname{Re} z$ sufficiently large, and these traces extend to meromorphic functions of $z$ for $\operatorname{Re} z>-1$ whose only singularities are simple poles at half-integer values of $z$. We use the notation $\{$ f.p.a. $z=0\}$ and $\lim _{z \rightarrow 0}$ to indicate the finite part at $z=0$ of these meromorphic continuations. In particular, $\{$ f.p.a. $z=0\}$ means that there is a potential pole at $z=0$, while $\lim _{z \rightarrow 0}$ means that there is no pole. 
The remaining operator traces in (2.6), (2.8), (2.9), are actually finite dimensional since $1-\partial_{y}\left(\partial_{y}^{\dagger} \partial_{y}\right)^{-1} \partial_{y}^{\dagger}$ and $\left(\partial_{y}^{\dagger} \partial_{y}\right)^{-1} \partial_{y}^{\dagger}-\left(\partial_{y_{0}}^{\dagger} \partial_{y}\right)^{-1} \partial_{y_{0}}^{\dagger}$ both vanish on the finite co-dimensional subspace $\operatorname{Im} \partial_{y}^{\dagger}$ of $L^{2}\left(X, S_{-} \otimes E\right)$.

It is easy to verify (see, e.g. Proposition (2.8) of [7]) that

$$
\begin{gathered}
d \ln \operatorname{det} \partial^{\dagger} \partial=\{\text { f.p.a. } z=0\} \operatorname{Tr}\left(\partial^{\dagger} \partial\right)^{-z-1}\left(\partial^{\dagger}(\nabla \partial)+\left(\nabla \partial^{\dagger}\right) \partial\right), \\
d \ln \operatorname{det} \partial_{y_{0}}^{\dagger} \partial=\{\text { f.p.a. } z=0\} \operatorname{Tr}\left(\partial_{y_{0}}^{\dagger} \partial\right)^{-z-1} \partial_{y_{0}}^{\dagger}(d \partial) .
\end{gathered}
$$

The results of [8] which we will need are summarized in the following Propositions.

\section{Proposition (2.12).}

$$
\frac{1}{2} d \ln \left(h_{0} \operatorname{det} \partial^{\dagger} \partial\right)+\sigma_{0}^{\theta}=\omega^{\theta}+z_{0}^{\theta}=d \ln \operatorname{det} \partial_{y_{0}}^{\dagger} \partial+J_{y_{0}}+L^{\theta}-\operatorname{Tr}\left(1-P_{y_{0}}\right) \theta .
$$

The second equality does not appear explicitly in [8]. However, it is immediate from the definitions and (2.11).

\section{Proposition (2.13).}

$$
\left.L^{\theta}=-\int_{X} \hat{A}\left(\mathscr{R}_{g}\right) \operatorname{tr} \theta \exp i \mathscr{F}_{\nabla^{E}} / 2 \pi\right)
$$

Theorem (2.14).

$$
d_{Y}\left(\sigma_{0}^{\theta}+\operatorname{Tr}\left(1-P_{y_{0}}\right) \theta\right)=2 \pi i\left\{\int_{X} \hat{A}\left(\mathscr{R}_{g}\right) \operatorname{ch}\left(\mathscr{\mathscr { F }}_{\nabla \bar{E}}\right)\right\}_{\text {\{two form }\}} .
$$

Finally, suppose $Y=\mathbb{R}$ and that conditions 1 and 2 of Sect. 1 are satisfied.

\section{Theorem (2.15).}

$$
\frac{1}{2 \pi i} \int_{\mathbb{R}} \sigma_{0}^{\theta}=\xi\left(\left.H\right|_{\bar{V}}\right) \bmod 1 .
$$

In [8] we interpreted $\omega^{\theta}+z_{0}^{\theta}$ geometrically in terms of the connection $\nabla^{(\mathscr{L})}$ on the determinant line bundle $\mathscr{L}$ of the family of operators $\partial$. In this context, Theorem (2.14) gives the curvature of $\nabla^{(\mathscr{L})}$, while Theorem (2.15) relates parallel transport in $\mathscr{L}$ relative to $\nabla^{(\mathscr{L})}$ with parallel transport in the trivial bundle $\operatorname{Ker} \partial_{-\infty}^{\dagger}$.

\section{Proof of Theorem (1.1)}

We now turn to the proof of Theorem (1.1). If we take $Y=\mathbb{R}, y_{0}=-\infty$, and integrate the formula of Proposition (2.12) using Theorem (2.15), we are reduced to proving

Proposition (3.1). There is a universal local functional B satisfying (1.4) such that if $Y=\mathbb{R}$ and conditions 1 and 2 are satisfied, then

$$
\int_{\mathbb{R}}\left(J_{-\infty}+L^{\theta}\right)=Q\left(\nabla^{\bar{E}}\right)+B\left(\nabla_{-\infty}^{E}, \nabla_{\infty}^{E}, g\right) .
$$

We begin by showing, for arbitrary $Y$, that $J_{y_{0}}$ is local. 
Proposition (3.2). There is an explicitly computable universal polynomial $\left[J_{y_{0}}\right]$ in $(\operatorname{det} g)^{-1}$, the components of $g$, and the components of the multiple covariant derivatives (in the $X$ directions) of $\mathscr{R}_{g}, \mathscr{F}_{\nabla_{y_{0}}^{E}}, \nabla^{E}-\nabla_{y_{0}}^{E}$, and $d_{Y} \nabla^{E}$ such that

$$
J_{y_{0}}=\int_{X}|d x|\left[J_{y_{0}}\right]\left(g, \nabla^{*}\left(\mathscr{R}_{g}\right), \nabla^{*}\left(\mathscr{F}_{\nabla_{y_{0}}}\right), \nabla^{*}\left(\nabla^{E}-\nabla_{y_{0}}^{E}\right), \nabla^{*}\left(d_{Y} \nabla^{E}\right) .\right.
$$

Here we view $\widetilde{\mathscr{F}}_{\nabla_{\mathcal{V}_{0}}^{E}}, \nabla^{E}-\nabla_{y_{0}}^{E}$, and $d_{Y} \nabla^{E}$ as elements of $\Omega^{*}(Y) \otimes \Omega^{*}(X$, End $E)$. Thus, for example,

$$
\nabla^{E}-\nabla_{y_{0}}^{E} \in \Omega^{0}(Y) \otimes \Omega^{1}(X, \text { End } E), \quad d_{Y} \nabla^{E} \in \Omega^{1}(Y) \otimes \Omega^{1}(X, \text { End } E) .
$$

Proof of Proposition (3.2). We use Propositions (C.6) and (C.8) of Appendix C of [7]. Observe that

$$
\begin{aligned}
& J_{y_{0}}=\lim _{z \rightarrow 0}-\operatorname{Tr} \int_{\mathscr{C}} \frac{d \lambda}{2 \pi i} \lambda^{-z} R(\lambda)-\operatorname{Tr} \int_{\mathscr{O}} \frac{d \lambda}{2 \pi i} R(\lambda), \\
& R(\lambda)=\lambda^{-1}\left(\left(\partial^{\dagger} \partial-\lambda\right)^{-1} \partial^{\dagger}-\left(\partial_{y_{0}}^{\dagger} \partial-\lambda\right)^{-1} \partial_{y_{0}}^{\dagger}\right) d \partial,
\end{aligned}
$$

where $\mathscr{C}$ is the contour in $C$ used to define the operator complex powers, and $\mathscr{O}$ is a small counterclockwise oriented circle in $C$ around 0 . It is easy to check that $R(\lambda)$ satisfies the hypothesis of Proposition (C.6) of [7]. In particular, the apparent $\lambda^{-1}$ pole in the complete symbol of $R(\lambda)$ is removable, and the complete symbol is analytic at $\lambda=0$. It thus follows from Proposition (C.6) that $J_{y_{0}}$ is expressible as the integral over $X$ of a density which in a local coordinate system is given by a local expression in the complete symbol of $R(\lambda)$. Now this symbol has the form

$$
\operatorname{tr} \sigma(R(\lambda))(\lambda, x, \xi)=\sum_{n, q . r} c_{n, q, r}(x, \xi) \lambda^{q}(a(x, \xi)-\lambda)^{-r},
$$

where $a(x, \xi)=g(x)_{i j} \xi^{i} \xi^{j}$ and $c_{n, q, r}(x, \xi)$ is a polynomial of degree $n-2 q+2 r$ in $\xi$ with $x$ dependent coefficients which depend polynomially on the derivatives at $x$ of the components of $g, \nabla_{y_{0}}^{E}, \nabla^{E}-\nabla_{y_{0}}^{E}$ and $d_{Y} \nabla^{E}$. As in [2], these derivatives can in turn be expressed in terms of covariant derivatives. The proof of the proposition is now completed by observing that by Proposition (C.8) of Appendix C of [7] the form (3.6) of $\sigma(R(\lambda))(\lambda, x, \xi)$ implies that $\left[J_{y_{0}}\right](x)$ is expressible as a polynomial in $(\operatorname{det} a)^{-1}$ and the coefficients of $c_{n, q, r}(x, \xi)$ and $a(x, \xi)$.

For notational convenience, given an arbitrary connection $\nabla^{\bar{E}}=d_{Y}+\theta+\nabla_{(\cdot)}^{E}$ on $\bar{E}=Y \times E$, we will write $\left[J_{y_{0}}\right]\left(\nabla^{\bar{E}}\right)$ for $\left[J_{y_{0}}\right]\left(g, \nabla^{*}\left(\mathscr{R}_{g}\right), \ldots, \nabla^{*}\left(d_{Y} \nabla^{E}\right)\right)$.

Now, for any two connections $\nabla^{1, E}$ and $\nabla^{2, E}$ on $E$ define

$$
B\left(\nabla^{1, E}, \nabla^{2, E}, g\right)=\int_{t \in[0,1]} \int_{X}|d x|\left[J_{v_{0}}\right]\left(\nabla^{[0,1] \times E}\right),
$$

where

$$
\nabla^{[0,1] \times E}=d t \frac{\partial}{\partial t}+t \nabla^{1, E}+(1-t) \nabla^{2, E} .
$$

Here we let $Y=[0,1]$ and view $\nabla^{[0,1] \times E}$ as connection on $[0,1] \times E$. It follows from the previous Proposition (3.2) that $B$ is a complex-valued functional of $\nabla^{1, E}, \nabla^{2, E}$, $g$, which is local in the sense of (1.4). 
Thus, to prove Proposition (3.1), it remains to show that the formula of the proposition holds with $B$ given by (3.7). In principle, this is an algebraic computation since $Q, L^{\theta}$, and $J_{y_{0}}$ are all expressible in terms of polynomials. We need "only" compute the polynomial $\left[J_{y_{0}}\right]$ and then perform some algebraic manipulations. Unfortunately, but as expected, this approach is hopelessly complicated, even if we restrict our attention to the imaginary part of $\left[J_{y_{0}}\right]$, which by Gilkey's theorem (see Sect. 4 below) is much simpler that the full $\left[J_{y_{0}}\right]$.

We thus proceed indirectly. The idea is that even though $J_{y_{0}}$ is difficult to compute, its exterior derivative $d_{Y} J_{y_{0}}$ can be calculated from Proposition (2.12) and Theorem (2.14). Then by Stoke's theorem, the line integral of $J_{y_{0}}$ along any path can be expressed in terms of the line integral along a fixed path, plus a surface integral of $d_{Y} J_{y_{0}}$. The relevant fixed path is the one that enters into the definition (3.7) of B; i.e. the linear path in the space of connections on $E$ from $\nabla_{-\infty}^{E}$ to $\nabla_{\infty}^{E}$. (It is this use of Stoke's theorem that requires us to enlarge the parameter manifold from $\mathbb{R}$ to $Y$.)

For an arbitrary connection $\nabla^{\bar{E}}$ on $\bar{E}$ define

$$
\tilde{J}_{y_{0}}^{\theta}\left(\nabla^{\bar{E}}\right)=\int_{X}|d x|\left[J_{y_{0}}\right]\left(\nabla^{\bar{E}}\right)-\int_{X} \hat{A}\left(\mathscr{R}_{g}\right) \operatorname{tr} \theta \exp i \mathscr{F}_{\nabla^{\bar{E}}} / 2 \pi .
$$

Thus $\widetilde{J}_{y_{0}}^{\theta}\left(\nabla^{\bar{E}}\right)$ is a one-form on $Y$ depending locally on $\nabla^{\bar{E}}$, and by Propositions (3.2) and (2.13),

$$
\widetilde{J}_{y_{0}}^{\theta}=J_{y_{0}}+L^{\theta}
$$

if the one-forms $J_{y_{0}}$ and $L^{\theta}$ are defined. Note, however, that while $J_{y_{0}}$ and $L^{\theta}$ are a priori defined only if $\partial_{y_{0}}^{\dagger} \partial_{y}$ is invertible, in contrast $\widetilde{J}_{y_{0}}^{\theta}\left(\nabla^{\bar{E}}\right)$ is defined for any connection without any conditions on the Weyl operators.

Proposition (3.10). For any connection $\nabla^{\bar{E}}$,

$$
d_{Y}\left(\widetilde{J}_{y_{0}}^{\theta}\left(\nabla^{\bar{E}}\right)\right)=2 \pi i\left[\int_{X} \hat{A}\left(\mathscr{R}_{g}\right) \operatorname{ch}\left(\mathscr{F}_{\nabla \bar{E}}\right)\right]_{\text {two form }} .
$$

This proposition is to be understood as a relationship between polynomials and as such could in principle be proved by algebraic manipulations. In the absence of an explicit expression for $\left[J_{y_{0}}\right]$, however, we must proceed indirectly.

Proof. For geometric data $\bar{E}, g, \nabla^{\bar{E}}$, etc. for which condition $1^{\prime}$ holds, the formula of the proposition follows by taking the exterior derivative of the formula of Proposition (2.12) and then using Theorem (2.14). This is enough to prove the Proposition in general, since the collection of such data is sufficiently abundant and the polynomial $\left[J_{y_{0}}\right]$ is universal.

We can now give the

Proof of Proposition (3.1). By hypothesis we are given a connection $\nabla^{\mathbb{R} \times E}$ on $\mathbb{R} \times E \rightarrow \mathbb{R} \times X$ satisfying conditions 1 and 2 of Sect. 1 . Let $Y=[0,1] \times \mathbb{R}$ and extend $\nabla^{\mathbb{R} \times E}$ to a connection $\nabla^{Y \times E}$ on $Y \times E \rightarrow Y \times X$ by setting

$$
\nabla^{Y \times E}=d t \frac{\partial}{\partial t}+(1-t) \nabla^{\mathbb{R} \times E}+t\left(d u \frac{\partial}{\partial u}+\nabla_{-\infty}^{E}\right), \quad(t, u) \in[0,1] \times \mathbb{R} .
$$


(Observe that $\nabla^{Y \times E}$ does not necessarily satisfy condition $1^{\prime}$.) By naturality it follows that

$$
\begin{gathered}
\left.\tilde{J}_{y_{0}}^{\theta}\left(\nabla^{Y \times E}\right)\right|_{1 \times \mathbb{R}}=0,\left.\quad \tilde{J}_{y_{0}}^{\theta}\left(\nabla^{Y \times E}\right)\right|_{0 \times \mathbb{R}}=\widetilde{J}_{y_{0}}^{\theta}\left(\nabla^{\mathbb{R} \times E}\right), \\
\left.\tilde{J}_{y_{0}}^{\theta}\left(\nabla^{Y \times E}\right)\right|_{[0,1] \times\{-\infty\}}=0,\left.\quad \tilde{J}_{y_{0}}^{\theta}\left(\nabla^{Y \times E}\right)\right|_{[0,1] \times\{\infty\}}=\tilde{J}_{y_{0}}^{\theta}\left(d t \frac{\partial}{\partial t}+(1-t) \nabla_{\infty}^{E}+t \nabla_{-\infty}^{E}\right) .
\end{gathered}
$$

Now, by Stoke's theorem

$$
\int_{0 \times \mathbb{R}} \widetilde{J}_{y_{0}}^{\theta}\left(\nabla^{Y \times E}\right)=\int_{[0,1] \times\{\infty\}} \widetilde{J}_{y_{0}}^{\theta}\left(\nabla^{Y \times E}\right)+\int_{[0,1] \times \mathbb{R}} d_{Y} \widetilde{J}_{y_{0}}^{\theta}\left(\nabla^{Y \times E}\right) .
$$

By (3.9) and (3.12) the left-hand side of (3.13) is $\int_{\mathbb{R}} J_{y_{0}}+L^{\theta}$; by (3.12) the first term on the right-hand side is $B\left(\nabla_{-\infty}^{E}, \nabla_{-\infty}^{E}\right)$; and by Proposition (3.10), the second term on the right-hand side is just $Q\left(\nabla^{\bar{E}}\right)$.

\section{The Counterterm B}

We conclude by giving some additional information about the local counterterm B.

Proposition (4.1). $[B]$ can be chosen so that $\operatorname{Im}[B]|d x|$ is the ring of local invariants generated by $\operatorname{tr} \mathscr{R}_{g}^{j}$ and $\operatorname{tr} m\left(\mathscr{F}_{\nabla_{-}{ }_{\infty}}, \nabla_{\infty}^{E}-\nabla_{-\infty}^{E}, D_{\nabla_{-}^{E}}\left(\nabla_{\infty}^{E}-\nabla_{-\infty}^{E}\right)\right)$.

Here $D_{V V_{y_{0}}}$ is the covariant exterior derivative on $\Omega^{*}(X$, End $E)$ determined by $\nabla_{-\infty}^{E}$. We view $\mathscr{F}_{\nabla_{-}}$and $\nabla^{E}-\nabla_{-\infty}^{E}$ as elements of the ring $\Omega^{*}(Y \times X$, End $E)$, and $m$ is a monomial in these quantities. $j$ is a non-negative integer and $\mathscr{R}_{g}^{j}$ is taken in the ring $\Omega^{*}(X$, End $T X)$.

Proof. By the generalized Gilkey theorem of Appendix B of [7] it suffices to show that the assignment

$$
\left(g, \nabla_{-\infty}^{E}, \nabla_{-\infty}^{E}-\nabla_{\infty}^{E}\right) \mapsto \operatorname{Im} B\left(\nabla_{-\infty}^{E}, \nabla_{\infty}^{E}, g\right)
$$

defines a weight zero, regular, form-valued invariant of the metric $g$, the connection $\nabla_{-\infty}^{E}$, and the endomorphism valued one-form $\nabla_{-\infty}^{E}-\nabla_{\infty}^{E}$. Naturality and regularity follow from Proposition (3.2). As for the weight, if $g$ is scaled by $\alpha$, then $\partial$ is scaled by $\alpha^{-1}$, and it follows from (3.4) and (3.5) that $J_{y_{0}}$ is unchanged. Thus by (3.7), $B$ is also unchanged. Finally $\operatorname{Im} B$ defines a differential form valued invariant (as opposed to just a measure) since under a change in orientation of $X, \operatorname{Im} B$ changes sign. In fact, up to bundle isomorphism, a change of orientation interchanges $S_{+}$and $S_{-}$and hence amounts to interchanging $\partial$ and $\partial^{\dagger}$. Then, up to cyclic permutation of operators, $R(\lambda)$ in definition (3.5) gets replaced by its adjoint, and thus $\operatorname{Im} J_{y_{0}}$ gets replaced by $-\operatorname{Im} J_{v_{0}}$.

In a similar fashion we can prove

Proposition (4.3). $\left[J_{y_{0}}\right]$ can be chosen so that $\operatorname{Im}\left[J_{y_{0}}\right]|d x|$ is a differential form of degree $(1, \operatorname{dim} X)$ on $Y \times X$ which is in the ring of invariants generated by $\operatorname{tr} \mathscr{R}_{g}^{j}$ and $\operatorname{tr} m\left(\mathscr{F}_{\nabla \bar{y}_{0}}^{E}, \nabla^{E}-\nabla_{y_{0}}^{E}, D_{\nabla_{y_{0}}^{E}}\left(\nabla^{E}-\nabla_{y_{0}}^{E}\right), d_{Y} \nabla^{E}, D_{V_{y_{0}}^{E}}\left(d_{Y} \nabla^{E}\right)\right)$. 
Now $m(\ldots)$ is a monomial in $\Omega^{*}(Y \times X$, End $E)$.

For $\operatorname{dim} X=4$, a tedious but straightforward calculation using the formulae of Propositions (C.6) and (C.17) of [7] shows that we can take

$$
\begin{gathered}
\operatorname{Im}\left[J_{y_{0}}\right]|d x|=\frac{1}{3 !} \frac{1}{(2 \pi)^{2}} 3 \operatorname{tr} v \\
\left(\frac{1}{2}\left(\mathscr{A}_{y_{0}} \mathscr{F}_{\nabla_{y_{0}}}+\mathscr{F}_{\nabla_{y_{0}}} \mathscr{A}_{y_{0}}\right)+\frac{1}{3}\left(\mathscr{A}_{y_{0}}\left(D_{\nabla_{y_{0}}} \mathscr{A}_{y_{0}}\right)+\left(D_{\nabla_{y_{0}}} \mathscr{A}_{y_{0}}\right) \mathscr{A}_{y_{0}}\right)+\frac{1}{2} \mathscr{A}_{y_{0}}^{3}\right),
\end{gathered}
$$

where $\mathscr{A}_{y_{0}}=\nabla^{E}-\nabla_{y_{0}}^{E}$ and $v=d_{Y} \nabla^{E}$.

This can be written more suggestively as

$$
\operatorname{Im}\left[J_{y_{0}}\right]|d x|=2 \pi \int_{s \in[0,1]}\left\{\operatorname{tr} \hat{A}\left(\mathscr{R}_{g}\right) \operatorname{ch}\left(\mathscr{F}_{d s \partial / o s}+d_{Y}+s \nabla E y+(1-s) \nabla^{E}\right)\right\}_{(1,1, \operatorname{dim} X)},
$$

where the subscript $\{1,1, \operatorname{dim} X\}$ indicates the grading in $\Omega^{*, *} *([0,1] \times Y \times X)$. We have verified (4.5) for $\operatorname{dim} X=2,4$ and conjecture that (4.5) formula holds in arbitrary dimensions.

When formula (4.5) holds, $\operatorname{Im} B\left(\nabla_{-\infty}, \nabla_{\infty}\right)$ vanishes. In fact, assuming (4.5), we have

$$
\begin{aligned}
\operatorname{Im} B & =\int_{X} \int_{t \in[0,1]} \operatorname{Im}\left[J_{y_{0}}\right]\left(d t \frac{\partial}{\partial t}+t \nabla_{-\infty}^{E}+(1-t) \nabla_{\infty}^{E}\right)|d x| \\
& =2 \pi \int_{X} \int_{t \in[0,1]} \int_{s \in[0,1]} \hat{A}(\mathscr{R}) \operatorname{ch}\left(\mathscr{F}_{d t \partial / \partial t+d s d / d s+\nabla \underline{-}_{\infty}+s t\left(\nabla_{\infty}^{E}-\nabla E_{\infty}\right)}\right) .
\end{aligned}
$$

Now if $\mu$ is the orientation reversing map of $[0,1] \times[0,1] \times X$ into itself which interchanges the first two factors, then $\int \alpha=-\int \mu^{*} \alpha$ for any differential form $\alpha$ on $[0,1] \times[0,1] \times X$. Since the integrand in (4.6) is invariant under $\mu^{*}$, its integral must vanish.

These observations prove Proposition (1.5).

Acknowledgements. We wish to thank R. Bott, M. Goulian, G. Moore, P. Nelson, and C. Taubes for valuable discussions.

\section{References}

1. Alvarez-Gaumé, L., Della Pietra, S., Della Pietra, V.: The effective action for chiral fermions. Phys. Lett. B (to appear) .

2. Atiyah, M.F., Bott, R., Patodi, V.K.: On the heat equation and the index theorem. Invent. Math. 19, 279 (1975)

3. Atiyah, M.F., Patodi, V.K., Singer, I.M.: Spectral asymmetry and Riemannian geometry. I, II, III. Math. Proc. Camb. Phil. Soc. 77, 43 (1975); 78, 405 (1975); 79, 71 (1976)

4. Atiyah, M.F., Singer, I.M.: Dirac operators coupled to vector potentials. Proc. Natl. Acad. Sci. 81, 2597 (1984)

5. Bismut, J.-M., Freed, D.: Geometry of elliptic families: anomalies and determinants. Preprint

6. Bismut, J.-M., Freed, D.: The analysis of elliptic families: metrics and connections on determinant bundles. Commun. Math. Phys. 106, 159 (1986). The analysis of elliptic families: Dirac operators, eta invariants, and the holonomy theorem. Commun. Math. Phys. 107, 103 (1986)

7. Della Pietra, S., Della Pietra, V.: Parallel transport in the determinant line bundle: the zero index case. Commun. Math. Phys. (to appear) 
8. Della Pietra, S., Della Pietra, V.: Parallel transport in the determinant line bundle: the nonzero index case Commun. Math. Phys. (to appear)

9. Gilkey, P.B., Smith, L.: The eta invariant for a class of elliptic boundary value problems. Commun. Pure and Appl. Math. 36, 85 (1983)

10. Kato, T.: Perturbation theory for linear operators. Berlin, Heidelberg, New York: Springer 1980

11. Lott, J.: Vacuum charge and the eta function. Commun. Math. Phys. 93, 533 (1984)

12. Reed, M., Simon, B.: Methods of modern mathematical physics, Vols. I, II, IV. New York, London: Academic Press 1978

13. Seeley, R.: Complex powers of an elliptic operator. In: Singular integrals. Proc. Sym. Pure Math. Vol. X. Am. Math. Soc. (1967)

14. Seeley, R.: Topics in pseudo differential operators. 1968 CIME Lectures. In: Pseudo differential operators. Edizioni Cremonese 1969

15. Witten, E.: An $S U(2)$ anomaly. Phys. Lett. 117B, 324 (1982)

16. Witten, E.: Global gravitational anomalies. Commun. Math. Phys. 100, 197 (1985)

Communicated by A. Jaffe

Received September 22, 1986 